\title{
HCV full-length genome reconstruction with sequence independent amplification combined with next generation sequencing
}

\author{
Barbara Bartolini", Emanuela Giombini, Isabella Abbate, Ubaldo Visco-Comandini, Gianpiero D'offizi, \\ Gabriella Rozera, Marina Selleri, Giuseppe Ippolito, Maria Rosaria Capobianchi \\ From 17th International Symposium on HIV and Emerging Infectious Diseases (ISHEID) \\ Marseille, France. 23-25 May 2012
}

\section{Introduction}

$\mathrm{HCV}$ genome variability is related to both disease progression and treatment response. De novo high-throughput pyrosequencing was used to obtain full length $\mathrm{HCV}$ genome characterization directly from clinical samples.

\section{Material and methods}

Plasma samples from $3 \mathrm{HCV}$-infected subjects were analyzed (two patients with subtype $1 \mathrm{~b}$, one patient with subtype $2 \mathrm{a} / 2 \mathrm{c}$; viral load: $6.0 \times 10^{6}, 20.8 \times 10^{6}$ and $7.3 \times 10^{6} \mathrm{IU} / \mathrm{ml}$ viral load, respectively). All samples were analyzed in a single run, using sample-specific barcoding adapters. Data were generated with a modified sequence-independent single primer amplification followed by 454 sequencing (GS-FLX Roche, Titanium version), using the shotgun approach. The reads were assembled using cap3 program; $\mathrm{HCV}$ contigs were identified using BLAST against full $\mathrm{HCV}$ genome database. Reads of HCV contigs were used for genome reconstruction with gs Mapper (Roche software).

\section{Results}

A total of 297,493 reads were obtained (average length 267 bp). Using a minimum read length cut off of $40 \mathrm{nt}$ with $>90 \%$ identity and $>40 \%$ overlapping, BLAST analysis classified a total of 27,107 reads $(10,682$ from patient $1,11,920$ from patient 2, and 4,505 from patient 3 ) as $\mathrm{HCV}$-specific. In all patients, genome reconstruction was achieved for more than $98 \%$ of the entire $\mathrm{HCV}$ genome. The mean coverage was 315, 307 and 142 reads per site for patients 1,2 and 3, respectively (overall mean coverage: 253 reads per site). Within-patient variability was calculated, resulting in E1 and E2 as the most variable structural genes in all patients, as expected.

\section{Conclusion}

The present study describes a unifying approach for $\mathrm{HCV}$ full genome sequencing, based on sequence-independent amplification combined with next generation sequencing. This may represent a relevant innovation, since so-far $\mathrm{HCV}$ full genome direct sequencing was based on genotype-specific multiple primer approach and conventional sequencing. The possibility of performing simultaneous analysis of pooled samples may represent a further advantage for cost saving.High coverage allows to analyze virus variability along the entire genome providing important information on possible viral variants which could impact on clinical and therapeutic outcome.

Published: 25 May 2012

doi:10.1186/1742-4690-9-S1-O6

Cite this article as: Bartolini et al:: HCV full-length genome

reconstruction with sequence independent amplification combined with next generation sequencing. Retrovirology 2012 9(Suppl 1):O6.

\footnotetext{
* Correspondence: barbara.bartolini@inmi.it

Virology at National Institute for Infectious Diseases L. Spallanzani, Rome, Italy
}

(c) 2012 Barbara et al; licensee BioMed Central Ltd. This is an Open Access article distributed under the terms of the Creative Commons 